\title{
INVESTIGACIÓN
}

Recibido: 19/06/2019 --- Aceptado: 20/12/2019 --- Publicado: 15/06/2020

\section{LA EXPERIENCIA COMUNITARIA DEL CONSUMO DE MARCAS. DE LA SUBCULTURA DE CONSUMO A LA TRIBU CONSUMIDORA}

\section{The community experience of brand consumption. From the subculture of consumption to the consumer tribe}

Paloma Sanz-Marcos¹. Universidad de Sevilla. España.

palomasanz@us.es

Rodrigo Elías-Zambrano. Universidad de Sevilla. España. rodrigoelias@us.es

\section{RESUMEN}

Existen numerosas taxonomías de consumidores que abordan el comportamiento de los mismos con respecto a la marca. No obstante, existe cierta confusión a la hora de distinguir y definir las características de los conceptos que se incluyen en estas clasificaciones. La principal novedad de este estudio radica en la sintetización de las principales aportaciones teóricas que estudian el comportamiento colectivo de los consumidores con objeto de explorar las implicaciones concretas que suponen para la gestión de marca. Para ello se exploran las diferencias conceptuales entre los términos subcultura de consumo, comunidad de marca y tribu consumidora con objeto de proponer un marco de referencia comparativo que relacione el compromiso hacia la marca, el sentido de pertenencia entre los consumidores y la construcción del significado de marca para los mismos. Los resultados indican que, en efecto, las diferencias conceptuales entre estos términos han de ser abordadas por los profesionales de la gestión de marca con objeto de implementar de manera eficaz las estrategias de branding.

PALABRAS CLAVE: comunidades de consumo - subculturas de consumo - tribus consumidoras - comunidades de marca - neotribus - branding.

\section{ABSTRACT}

There are numerous consumer taxonomies that address their behavior with respect to the brand. However, there is some confusion in distinguishing and defining the

\footnotetext{
${ }^{1}$ Paloma Sanz-Marcos: profesora del departamento de Comunicación audiovisual y Publicidad de la Universidad de Sevilla. Doctora en Comunicación (mención internacional por la UC Berkeley, California) y licenciada en Publicidad y Relaciones Públicas. palomasanz@us.es
} 
characteristics of the concepts included in these classifications. The main novelty of this study lies in the synthesis of the main theoretical contributions that study the collective behavior of consumers in order to explore the specific implications that they entail for brand management. For this, the conceptual differences between the terms subculture of consumption, brand community and consumer tribe are explored in order to propose a comparative frame of reference that relates the commitment to the brand, the sense of belonging among consumers and the construction of brand meaning for them. The results indicate that, in effect, the conceptual differences between these terms have to be addressed by brand management professionals in order to effectively implement the strategies of branding.

KEYWORDS: consumer communities - subcultures of consumption - consumer tribes - brand communities - neotribes - branding.

\section{A EXPERIÊNCIA COMUNITÁRIA DO CONSUMO DE MARCA. DA SUBCULTURA DE CONSUMO À TRIBO DO CONSUMIDOR}

\section{RESUMO}

Existem numerosas taxonomias de consumidores que abordam o comportamento dos mesmos com respeito a marca. Não obstante, existe certa confusão na hora de distinguir e definir as características dos conceitos que se incluem nestas classificações. A principal novidade deste estudo radica na sintetização das principais aportações teóricas que estudam o comportamento coletivo dos consumidores com a finalidade de explorar as implicações concretas que supõem para o gerenciamento da marca. Para isto se exploram as diferenças conceituais dentre os termos subcultura de consumo, comunidade de marca e tribo do consumidor com o objetivo de propor uma estrutura de referência comparativa que relacione o compromisso com a marca, o sentimento de pertence entre os consumidores e a construção do significado de marca para eles. Os resultados indicam que, de fato, as diferenças conceituais entre estes termos devem ser abordadas pelos profissionais da gestão de marca com o objetivo de implementar de forma eficaz as estratégias de branding.

PALAVRAS CHAVE: comunidades de consumo - subculturas de consumo - tribo do consumidor - comunidades de marca - neotribus - branding.

\section{Cómo citar el artículo:}

Sanz-Marcos, P. y Elías-Zambrano, R. (2020). La experiencia comunitaria del consumo de marcas. De la subcultura de consumo a la tribu consumidora. [The community experience of brand consumption. From the subculture of consumption to the consumer tribe].Vivat Academia. Revista de Comunicación, (151), 69-83. doi: http:// doi.org/10.15178/va.2020.151.69-83 Recuperado de

http://www.vivatacademia.net/index.php/vivat/article/view/1202 
Sanz-Marcos, P. y Elías-Zambrano, R.

La experiencia comunitaria del consumo de marcas. De la subcultura de consumo a la tribu consumidora

\section{INTRODUCCIÓN}

El mercado evoluciona hacia una sofisticación en la que se advierte un escenario donde las marcas se configuran como un valor estratégico fundamental (Fernández Gómez, 2013). Las marcas han ido adquiriendo una gran transformación manifestada en un mayor interés en la construcción de mensajes que dialoguen fluidamente con el universo social del consumidor. Concretamente, nos encontramos ante un nuevo prisma comunicativo en el que la comunidad toma su papel más relevante a la hora de entender a los consumidores. El poder de las comunidades en el mercado no puede subestimarse debido a que los vínculos sociales entre los sujetos proporcionan valor y recursos importantes que permiten construir la identidad e influir en las elecciones de consumo de éstos (Närvänen, Kartastenpää \& Kuusela, 2013, p. 358).

Para algunos autores este escenario se ve afectado por muchos de los cambios sociales que se han venido desarrollando bajo la etiqueta de posmodernidad, un movimiento social caracterizado por una hegemonía del consumo que provoca que instituciones como la familia, el lugar de trabajo, la comunidad o la iglesia, hayan perdido su legitimidad (Firat \& Venkatesh, 1993, p. 228). Este movimiento presenta a un consumidor afectado por una sociedad que presenta un marcado carácter fragmentado (Firat, Dholakia \& Venkatesh, 1995) en el que el individuo cuenta cada vez con menos apoyos o vínculos sociales en los que auxiliarse (cfr. Cova, 1997, p. 300).

Ante esta perspectiva, el sujeto busca en el consumo ese apoyo que las instituciones solían aportarle propiciando el acercamiento del individuo al consumo para definir su identidad (Cova, 1997; Kozinets, 1999). Así lo entienden autores como Drawbaugh, quienes afirman que el declive que instituciones tradicionales como la iglesia, la familia y la comunidad están sufriendo en algunas partes del mundo propicia que un creciente número de personas acuda a las marcas comerciales para definir su identidad personal (cfr. Drawbaugh, 2001, p. 5).

En consecuencia, se observa una necesidad por parte de los individuos de compensar esa falta de referentes a través del consumo. Uno de los modos en los que se manifiesta esa compensación es en la agrupación de los consumidores en torno a lo que se denominan comunidades de consumo (Canniford, 2011, p. 58). Estas agrupaciones comportan importantes implicaciones para el estudio de las marcas en tanto a que muestran cómo el consumo social mejora la naturaleza utilitaria de un producto o servicio gracias al valor que se obtiene de su conexión con una comunidad de usuarios (Mathwick, Wiertz \& De Ruyter, 2007, p. 832).

A pesar de que estas comunidades de consumo son de vital importancia para abordar el desarrollo teórico referente al comportamiento del consumidor, las implicaciones que dichas agrupaciones tienen para el brand management y la necesidad de distinguir entre las distintas categorías de comunidades no ha sido 
Sanz-Marcos, P. y Elías-Zambrano, R.

La experiencia comunitaria del consumo de marcas. De la subcultura de consumo a la tribu consumidora

atendida con rigurosidad por parte del mundo académico. En este sentido, se sugiere que existe una carencia de descripciones consistentes y consensuadas en torno a las diferentes formas de comunidad.

\section{OBJETIVOS}

Este trabajo se plantea un doble objetivo. En primer lugar, persigue sintetizar las aportaciones teóricas que estudian el comportamiento grupal de los consumidores con el objeto de explorar las implicaciones concretas que suponen para la gestión de marca. En segundo lugar y de manera más específica, se propone analizar las diferencias conceptuales que existen entre los términos hallados con objeto de proponer un marco de referencia comparativo que relacione el compromiso hacia la marca, el sentido de pertenencia entre los consumidores y la construcción del significado de marca para los mismos.

\section{METODOLOGÍA}

Dado el carácter teórico de este trabajo, se ha acudido al metaanálisis bibliográfico, el cual se ha desarrollado a través de un estudio longitudinal. En este caso, el estudio se ha ocupado de analizar el fenómeno desde el año 1995, fecha de publicación del primer artículo científico dedicado al estudio del comportamiento grupal de los consumidores en relación a la gestión de marca, hasta la actualidad. Dicho criterio temporal ha permitido obtener tanto una información transversal del objeto de estudio, como una visión holística del concepto ya que, de otra forma, no se hubiese podido observar la evolución del fenómeno en conjunto con otros términos aparecidos a lo largo del tiempo así como el modo en el que se interrelacionan sus características e implicaciones.

En la tabla 1 se exponen brevemente los principales autores (si bien la lista es más extensa, como se puede comprobar en las referencias finales de este artículo) que se han revisado.

Tabla 1. Relación de autores analizados.

\begin{tabular}{|c|c|c|c|}
\hline AUTORES & AÑ & PUBLICACIÓN & CONCEPTO \\
\hline Cova \& Cova & 2002 & European Journal of Marketing & Tribu consumidora [Consumer tribe] \\
\hline Kozinets & 2007 & Routledge (monográfico) & Innotribe \\
\hline $\begin{array}{c}\text { Goulding \& } \\
\text { Shankar }\end{array}$ & 2011 & Annals of Tourism Research & $\begin{array}{c}\text { Neo-tribu } \\
\text { [Neotribe] }\end{array}$ \\
\hline Canniford & 2011 & Research in Consumer Behavior & $\begin{array}{c}\text { Comunidades de consumo } \\
\text { [Consumption communities] }\end{array}$ \\
\hline $\begin{array}{c}\text { Price, Thomas, } \\
\text { Schau }\end{array}$ & 2013 & Journal of Consumer Research & $\begin{array}{c}\text { Comunidades de consumo } \\
\text { [Consumption communities] }\end{array}$ \\
\hline $\begin{array}{c}\text { Bazaki \& } \\
\text { Veloutsou }\end{array}$ & 2010 & $\begin{array}{c}\text { ATINER } \\
\text { (monográfico) }\end{array}$ & $\begin{array}{c}\text { Neo-tribu [Neotribe] } \\
\text { Richardson }\end{array}$ \\
\hline
\end{tabular}


Sanz-Marcos, P. y Elías-Zambrano, R.

La experiencia comunitaria del consumo de marcas. De la subcultura de consumo a la tribu consumidora

\begin{tabular}{|c|c|c|c|}
\hline $\begin{array}{c}\text { Biraghi, } \\
\text { Gambetti \& } \\
\text { Pace }\end{array}$ & 2018 & Journal of business research & $\begin{array}{c}\text { Tribu consumidora } \\
\text { [Consumer tribe] }\end{array}$ \\
\hline $\begin{array}{c}\text { Mamali, Nuttal } \\
\text { \& Shankar }\end{array}$ & 2018 & Marketing Theory & $\begin{array}{c}\text { Tribu consumidora } \\
\text { [Consumer tribe] }\end{array}$ \\
\hline Taute \& Sierra & 2014 & $\begin{array}{c}\text { Journal of Product E Brand } \\
\text { Management }\end{array}$ & $\begin{array}{c}\text { Tribu antropológica de consumo } \\
\text { [Anthropological consumption tribe] }\end{array}$ \\
\hline $\begin{array}{c}\text { Muñiz \& } \\
\text { O'Guinn }\end{array}$ & 2001 & Journal of Consumer Research & $\begin{array}{c}\text { Comunidad de marca [Brand } \\
\text { community] }\end{array}$ \\
\hline $\begin{array}{c}\text { Thompson \& } \\
\text { Throester }\end{array}$ & 2002 & Journal of Consumer Research & $\begin{array}{c}\text { Microculturas de consumo } \\
\text { [Microcultures of consumption] }\end{array}$ \\
\hline $\begin{array}{c}\text { Schouten \& } \\
\text { McAlexander }\end{array}$ & 1995 & Journal of Marketing & $\begin{array}{c}\text { Subculturas de consumo [Subcultures } \\
\text { of consumption] }\end{array}$ \\
\hline Kett & 1999 & Sociology & Neo-tribu [Neotribe] \\
\hline $\begin{array}{c}\text { Thomas, Price \& } \\
\text { Schau }\end{array}$ & 2013 & Journal of Consumer Research & $\begin{array}{c}\text { Comunidades de } \\
\text { consumo[Consumption Communities] }\end{array}$ \\
\hline
\end{tabular}

Fuente: elaboración propia.

\section{DISCUSIÓN DE LOS RESULTADOS}

\subsection{Resultados de la transferencia del consumo al estudio de las agrupaciones}

El examen de la literatura analizada relativa a la descripción de la organización de los consumidores en torno a grupos y su relación con las marcas, revela una ambigüedad significativa que se manifiesta en la determinación de las características que definen y diferencian a cada tipo de colectivo (Thomas, Price \& Schau, 2013, p. 1012). Existen varios términos propios de la literatura mercadotécnica que ofrecen cierta confusión conceptual y que nos parece fundamental esclarecer para aproximarnos al objetivo de este trabajo.

Si bien el concepto de "comunidad" es una condición ineludible para entender los conceptos que abordan el consumo colectivo desde una perspectiva de marca, es en este aspecto donde prolifera la confusión terminológica resultando en un verdadero solapamiento de términos similares en cuanto al consumo colectivo (Thomas, Price \& Schau, 2013, p. 1012). A tales efectos, hemos recapitulado aquellos conceptos que más se repiten y ofrecen mayor confusión a lo largo de la literatura académica esto es, la subcultura de consumo, la comunidad de marca y la tribu consumidora.

\subsection{Las agrupaciones subversivas de consumidores: la subcultura de consumo}

En 1995 los autores Schouten \& McAlexander aportan uno de los estudios más completos acerca del consumo colectivo a través de las denominadas subculturas de consumo. En su artículo publicado en el Journal of Consumer Research titulado: "Subcultures of Consumption: An Ethnography of the New Bikers" (1995), analizan a fondo el término partiendo de la siguiente definición:

$\mathrm{T}$ We define a subculture of consumption as a distinctive subgroup of society that self-selects on the basis of a shared commitment to a particular product 
Sanz-Marcos, P. y Elías-Zambrano, R.

La experiencia comunitaria del consumo de marcas. De la subcultura de consumo a la tribu consumidora

class, brand, or consumption activity. Other characteristics of a subculture of consumption include identifiable, hierarchical social structure; a unique ethos, or set of shared beliefs and values; and unique jargons rituals, and modes of symbolic expression (1995, p. 43).

A través de esta definición, los investigadores manifiestan que el concepto encierra un importante carácter sociológico que identifica a un "subgrupo" de la sociedad con una serie de condicionantes únicos que los traslada al consumo.

Es por ello que, a la hora de entender el concepto de subcultura de consumo, es necesario reconocer que, por un lado, se trata de un término que bebe directamente de la sociología y que se erige como un agrupamiento alternativo a otras opciones sociales, y que, por otro lado, considera a un grupo de consumidores que comparten cierto compromiso respecto a una marca. Asimismo, otra de las características que nos ayudan a distinguir este concepto es el carácter subversivo que encierran estas subculturas de consumo. En el artículo anteriormente mencionado, Schouten \& McAlexander destacan que, como resultado de su estudio etnográfico, existe cierto grado de marginalidad y subversión (cfr. 1995, p. 48). Los miembros de la comunidad que ellos denominan como "HDSC" (Harley-Davidson-oriented subculture of consumption) ofrecen una serie de comportamientos que los distinguen de otras formas de agrupamiento en su interés por responder de forma alternativa a lo establecido (Bazaki \& Veloutsou, 2010, p. 163).

De este modo, entre los integrantes de las subculturas de consumo existe cierto interés por la marginalidad, por el rechazo a lo instaurado y por la experimentación de la diferencia respecto a los demás. Así lo entienden De Burgh-Woodman \& BraceGovan cuando afirman que estos grupos comparten creencias y experiencias que los sitúan aparte del resto, y cómo, incluso en ciertas ocasiones, pueden llegar a ser percibidos como contestatarios a las autoridades establecidas (cfr. 2007, p. 197). En efecto, esta particularidad es precisamente una de las características que de forma más clara exponen las diferencias existentes entre las subculturas de consumo y otro tipo de agrupaciones.

Asimismo, este rasgo subversivo manifiesta la estrecha relación sociológica que ya advertimos acerca del concepto. El hecho de que se encierre bajo la categoría de "subcultura" indica que se trata de un agrupamiento con un "modo de vida" concreto que no puede ser restringido a los límites de una comunidad de consumo" (cfr. Bazaki \& Veloutsou, 2010, p. 163).

Por último, otra de las características que alejan a las subculturas de consumo de otros conceptos, es el carácter jerárquico de las primeras. En este sentido, Schouten \& McAlexander, sostienen que "each subgroup within the HDSC maintains a formalhierarchy of officers" (1995, p. 49) y avanzan en la idea de que el estatus entre miembros se confiere "according to their seniority, participation and leadership ingroup activities, riding expertise and experience" (Schouten \& McAlexander, 1995,

Vivat Academia. Revista de Comunicación. 15 junio 2020 /15 septiembre 2020, nº 151, 69-83 
Sanz-Marcos, P. y Elías-Zambrano, R.

La experiencia comunitaria del consumo de marcas. De la subcultura de consumo a la tribu consumidora

p. 49), algo que una gran mayoría de académicos no encuentra en otros conceptos como las comunidades de marca o las tribus consumidoras.

Con todo esto, resulta paradójico que la confusión conceptual respecto a la subcultura de consumo afecte a otros conceptos. Este hecho nos resulta de especial importancia, dado que el concepto de comunidad de marca está comúnmente vinculado al de tribu consumidora. Por su parte, Woodman \& Brace-Govan tratan de explicar el motivo de dicha confusión, ofreciendo un punto de vista que entiende que el concepto de comunidad de marca proviene de una evolución en el estudio de las subculturas de consumo:

Yet, in recent years, the meaning of subculture in marketing discourse has been encroached upon by the concept of consumption, hence giving rise to the phrase «subculture of consumption», which in turn has spawned the study of «brand community» (2007, p. 193).

Por ello, haremos a continuación un esbozo de lo que se entiende en la literatura académica por comunidad de marca y las diferencias que comporta respecto a la tribu consumidora.

\subsection{Colectivos de consumidores devotos: la comunidad de marca}

El término comunidad de marca es acuñado por Albert Muñiz \& Thomas O'Guinn, quienes en el artículo "Brand Community" (2001), publicado en el Journal of Consumer Research, ofrecen la siguiente definición: "A brand community is a specialized, nongeographically bound community, based on a structured set of social relationships among admirers of a brand" (2001, p. 412). Este artículo, en el que asientan las bases del concepto, es la obra más citada en torno a las comunidades de marca y del que parten autores como Veloutsou y Moutinho para proponer su definición: "A brand community is as an enduring, self-selected group of consumers, sharinga system of values, standards and representations, who accept andrecognize bonds of membership with each other and with the whole" (2009, p. 316).Igualmente, encontramos el caso de McAlexander, Schouten \& Koenig, quienes también ofrecen su particular visión acerca de la comunidad de marca: "We take the perspective that brand community iscustomer-centric, that the existence and meaningfulness of the community in here in customer experience rather than in the brand around which that experience revolves"(2002, p. 39). Siguiendo la definición original de Muñiz \& O'Guinn, que ha servido de marco común a la gran mayoría de investigadores, es reseñable apuntar que existen varias características que nos ayudarán a conocer en mayor profundidad el concepto. Muñiz \& O'Guinn entienden que la comunidad de marca:

Like other communities, it is marked by a shared consciousness, rituals and traditions, and a sense of moral responsibility. Each of these qualities is, however, situatedwithin a commercial and mass-mediated ethos, and has its own particular expression. Brandcommunities are participants in the brand's larger social construction and play a vital role in thebrand's ultimate legacy (2001, p. 412).

Vivat Academia. Revista de Comunicación. 15 junio 2020 /15 septiembre 2020, n 151, 69-83 
Sanz-Marcos, P. y Elías-Zambrano, R.

La experiencia comunitaria del consumo de marcas. De la subcultura de consumo a la tribu consumidora

Así, existen tres rasgos que integran la definición de comunidad de marca: consciusness of a kind, el carácter ritual, y el sentido de responsabilidad moral (cfr. McAlexander, Schouten \& Koening, 2002, p. 42). En primer lugar, Muñiz \& O'Guinn entienden que la comunidad de marca está caracterizada por una conciencia compartida que hace referencia al sentido de pertenencia respecto al grupo. Así lo entienden cuando afirman que esta conciencia alude a la conexión intrínseca "that members feeltoward one another, and the collective sense of difference from others not in thecommunity. Consciousness of kind is shared consciousness, a way of thinking aboutthings that is more than shared attitudes or perceived similarity [...]" (Muñiz \& O'Guinn, 2001, p. 413). En efecto, se trata de una conciencia y forma de pensar compartida por los miembros de la comunidad, que al mismo tiempo concibe un sentimiento de diferencia respecto a aquellos que no pertenecen al grupo.

Al mismo tiempo, los autores avanzan que la manera de perpetuar esa conciencia se lleva a cabo a través de los rituales. Este carácter ritual, además de representar el segundo rasgo característico que encierran las comunidades de marca, manifiesta los procesos vitales en los que la comunidad es reproducida y transmitida. Cada vez que uno de los rituales es iniciado, los miembros de la comunidad son validados en su conocimiento de la comunidad (cfr. Muñiz \& O'Guinn, 2001, p. 421).

En cuanto al tercer rasgo, el de responsabilidad moral (sense of moral responsibility), alude al "sense of duty or obligation to the Communityas a whole, and to its individual members" (Muñiz \& O'Guinn, 2001, p. 413) y destaca por el deber moral que comparten sus miembros debido a un resultado que, entendemos, se origina de forma natural, como consecuencia de las dos primeras características. En este sentido, partimos de la idea de que, si existe una conciencia compartida que se refleja en una serie de prácticas sociales - que a su vez integran una serie de valores entre los miembros del grupo-irremediablemente se desarrollará una responsabilidad moral.

No obstante, la idea más relevante que se desprende para nuestro estudio hace referencia a la hegemonía que la marca supone para esa comunidad. En su artículo, Muñiz \& O'Guinn sostienen que la admiración por una determinada marca es de hecho la clave para ser reconocido como miembro de una determinada comunidad de marca:

members also frequently note a critical demarcation between users of their brand and users of other brands. There is some important quality, not always easily verbalized, that sets them apart from others and makes them similar to one another. Such a demarcation usually includes a reference to brand users being «different» or «special» in comparison to users of other brands (2001, p. 418).

La devoción por una determinada marca apoya en gran medida el sentido de pertenencia que los miembros de esa determinada "comunidad de marca" pueden llegar a incorporar. De hecho, los autores reconocen que "[...] the shared consciousness of brand communities is also informed by an explicitly commercial 
Sanz-Marcos, P. y Elías-Zambrano, R.

La experiencia comunitaria del consumo de marcas. De la subcultura de consumo a la tribu consumidora

and competitive marketplace ethos (e.g., Coke vs. Pepsi)" (Muñiz \& O'Guinn, 2001, p. 419). De modo que el sentimiento que los miembros de la comunidad sienten por Pepsi es justo lo que los diferencia de la comunidad de miembros que admiran a Coca-Cola, y a su vez lo que los hace partícipes de esa conciencia compartida de pertenecer a una misma comunidad.

\subsection{El vínculo emocional como aglutinador de consumidores: la tribu consumidora}

Uno de los desarrollos recientes en la literatura académica sobre los distintos colectivos de consumidores son las llamadas tribus consumidoras. Este concepto se desarrolla a partir de 1999, cuando el académico Bernard Cova destaca un enfoque alternativo al marketing relacional, tradicionalmente propulsado por pensadores norteamericanos, denominado como marketing tribal que persigue adecuarse a las exigencias del mercado mediterráneo. Esta corriente se presenta como una nueva perspectiva que permite entender las relaciones entre los consumidores (Tuominen, 2011). Las tribus consumidoras, se entienden como el principal agente de este enfoque basándose, principalmente, en el reconocimiento de la importancia del establecimiento de los vínculos emocionales entre los individuos por encima del consumo del propio producto (Silva \& Santos, 2012). Estas tribus tienen considerables implicaciones para el consumo y, más concretamente, para el estudio de la marca. Uno de sus rasgos más significativos con respecto a estas implicaciones es el enfoque de las estrategias del marketing tribal hacia la creación de una red de personas cuyo principal objetivo es hallar la interacción social alrededor de las marcas (Saat, Maisurah \& Hanim, 2015).

Es preciso destacar que la perspectiva tradicional del marketing, el cual comprende un intercambio diádico entre la organización y el consumidor, pierde importancia en este caso. En su lugar, la perspectiva tribal aboga por un acercamiento entre los consumidores subordinando el protagonismo de la marca a la relación entre éstos. La marca apoya la relación entre los consumidores y actúa como enlace entre los individuos (Dahl, 2014). Esta perspectiva se enfoca al estudio del consumidor entendiendo a éste como un agente verdaderamente activo en el consumo, destacando así su poder en las decisiones estratégicas de la marca. Así, esta corriente entiende que el valor es creado por los propios consumidores a los que se considera como agentes integrados en el proceso de marketing con capacidad para contribuir explícitamente a la creación de valores para las marcas (cfr. Cova \& Dalli, 2008).

Asimismo, las tribus consumidoras difieren de otros conceptos anteriormente comentados como las comunidades de marca en un rasgo fundamental: el aspecto que la marca adquiere para sus miembros. Si para estas comunidades la hegemonía de la marca es su principal insignia, en el caso de las tribus consumidoras son las relaciones entre sus miembros las que cobran mayor importancia (cfr. Dahl, 2014, p. 21).

Del mismo modo lo entienden Goulding, Shankar \& Canniford cuando expresan que: "[...] unlike brand communities tribes do not seek iconic brands as loci for

Vivat Academia. Revista de Comunicación. 15 junio 2020 /15 septiembre 2020, nº 151, 69-83 
Sanz-Marcos, P. y Elías-Zambrano, R.

La experiencia comunitaria del consumo de marcas. De la subcultura de consumo a la tribu consumidora

consumption experiences. On the contrary, within tribes, the social links established between consumers $[\ldots]$ are more important than whatever is being consumed (Cova, 1997)" (2013, p. 815).

Por su parte, Cova \& Cova sentencian que las "brand communities are explicitly commercial, whereas tribes are not" (Cova \& Cova, 2002, p. 603), apoyando igualmente esta idea. En cuanto a la responsabilidad moral que según Muñiz \& O'Guinn caracteriza a las comunidades de marca, es otro rasgo que aleja este concepto del de tribu consumidora. Esta responsabilidad moral responde al sentido de pertenencia que manifiestan estas comunidades. Como advierten los autores: "The sense of moral responsibility is what produces collective action and contributes to group cohesion. Moral responsibility need not be limited to punitive strictures concerning life and death matters, but rather every day, but nonetheless important, social commitments"' (Muñiz \& O'Guinn, 2001, p. 242).

En efecto, esta responsabilidad alude a un cierto grado de compromiso que, a su vez, permite la perpetuación y mantenimiento de la comunidad. Al mismo tiempo, las tribus consumidoras expresan un menor grado de implicación que como advierten Muñiz \& O'Guinn se distingue de las comunidades de marca en que éstas son

less ephemeral and their members as more committed than the ones [consumer tribes] described by Cova (1997) or Maffesoli (1996) [...] Brand communities can be relatively stable groupings, with relatively strong (but rarely extreme) degrees of commitment. Their moral responsibility may be a limited and subtle one, but it is a nontrivial one (Maffesoli, 1996) (Muñiz \& O'Guinn, 2001, p. 415).

Por su parte, Bazaki \& Veloutsou sostienen que este carácter efímero que poseen las tribus parece ser consecuencia de los lazos sociales que marcan a sus miembros. A tales efectos, los autores avanzan que

Brand communities being more stable, centralised, hierarchical whose members exhibit relatively high levels of commitment whereas tribes rely on the emotional bond between their members rather than on centralised power [the brand] for their existence and therefore are more fluid, fuzzy and informal (2010, p. 172).

De manera que, al contrario de lo que ocurre en las comunidades de marca, la delimitación de la tribu urbana ofrece un carácter más difuso que puede incluso manifestarse en la pertenencia múltiple y simultánea de sus miembros a otras tribus. Así lo expresan Goulding, Shankar \& Canniford cuando advierten que "[...] membership of one kind of tribe does not preclude membership from other tribes or communities" (Goulding, Shankar \& Canniford, 2013, p. 815), dato que, como venimos comentando no se presenta en la pertenencia a las comunidades de marca.

Cabe destacar que las investigaciones más recientes ofrecen una perspectiva que parece abrir paso a una concepción de la tribu adaptada a escenarios que escapan de la exclusividad de la marca como es caso el ámbito empresarial, donde autores como

Vivat Academia. Revista de Comunicación. 15 junio 2020 /15 septiembre 2020, nº 151, 69-83 
Sanz-Marcos, P. y Elías-Zambrano, R.

La experiencia comunitaria del consumo de marcas. De la subcultura de consumo a la tribu consumidora

Mamali, Nuttal \& Shankar (2018) plantean su carácter híbrido a la hora de participar en los mercados. Algo que a su vez recogen Biraghi, Gambetti \& Pace (2018) en el caso concreto del entorno online cuando plantean que las tribus consumidoras se consideran como apoyos sociales que permiten mejorar las capacidades de los consumidores a la hora de emprender en proyectos empresariales. En este sentido, señalan cómo el carácter emprendedor del consumidor logra beneficiarse de un esfuerzo tribal común para obtener ventajas en los mercados.

En definitiva, las tribus consumidoras ofrecen importantes implicaciones respecto a la marca que, siguiendo a Cova, Kozinets y Shankar (2007), no suponen una nueva forma de organización, sino una nueva forma de pensar acerca de los problemas de la organización.

\section{CONCLUSIONES}

La comunidad empresarial a menudo se ha centrado en cómo utilizar las interacciones sociales de los consumidores para lograr óptimos resultados de marketing. Concretamente, el estudio del comportamiento del consumidor desde un punto de vista social es una tarea que, unida al estudio del branding, se presenta notablemente compleja. A pesar de que el interés académico por entender la forma en la que los consumidores se relacionan con una marca es una tendencia destacable en la actualidad, se aprecia que es necesario seguir investigando para aportar conocimientos demostrables que describan rigurosamente la realidad de los sujetos.

La revisión de la literatura académica realizada para esta investigación ha permitido deducir que los conceptos de comunidad de marca, subcultura de consumo y tribu consumidora son aquellos que gozan de mayor notoriedad entre los académicos a la hora de agrupar a los consumidores por colectivos, siendo, al mismo tiempo, términos que se encuentran alejados conceptualmente entre sí como se puede observar en la tabla 2 .

Tabla 2. Diferencias entre los conceptos de subcultura de consumo, comunidad de marca y tribu consumidora.

\begin{tabular}{|c|c|c|}
\hline $\begin{array}{c}\text { SUBCULTURA DE } \\
\text { CONSUMO }\end{array}$ & $\begin{array}{c}\text { COMUNIDAD DE } \\
\text { MARCA }\end{array}$ & CONSUMIDORA \\
\hline $\begin{array}{c}\text { Compromiso hacia una } \\
\text { marca }\end{array}$ & $\begin{array}{c}\text { Compromiso hacia una } \\
\text { marca }\end{array}$ & $\begin{array}{c}\text { No compromiso hacia } \\
\text { una marca }\end{array}$ \\
\hline Estructura jerárquica & - & - \\
\hline Valores marginales & - & - \\
\hline- & Consciusness of a kind & $\begin{array}{c}\text { Efímeras y pertenencia } \\
\text { múltiple }\end{array}$ \\
\hline
\end{tabular}

Fuente: elaboración propia. 
Sanz-Marcos, P. y Elías-Zambrano, R.

La experiencia comunitaria del consumo de marcas. De la subcultura de consumo a la tribu consumidora

Las razones fundamentales que justifican esta conclusión se basan, principalmente en que, por un lado, a diferencia de la subcultura de consumo, la tribu consumidora y la comunidad de marca no se presentan como agrupaciones marginales o apartadas de la cultura dominante y con un marcado corte subversivo y, por otro y con respecto a la comunidad de marca, los miembros de las tribus consumidoras y las subculturas de consumo no presentan una evidente lealtad hacia la marca. Esta percepción, cuestiona la perspectiva de otros autores estudiados que, paradójicamente, encuentran ciertas similitudes conceptuales entre dichos términos, algo que, en efecto, pone en relieve la importancia de este estudio y la necesidad de ampliarlo en investigaciones ulteriores.

En lo relativo a las implicaciones concretas con respecto a la marca, se manifiesta que el estudio del comportamiento del consumidor a través de colectivos facilita el abordaje y aplicación de estrategias de branding efectivas. El reconocimiento de pautas similares por parte de los consumidores con respecto a las marcas pone de manifiesto que, en efecto, es posible implementar estrategias concretas de una manera eficiente. No obstante, se advierten importantes consecuencias a la hora de identificar a estos colectivos por parte del mundo profesional. Si bien los académicos pueden no llegar a reconocer con exactitud las diferencias conceptuales entre los términos analizados, es cierto que los gestores de marca pueden igualmente encontrar dificultades a la hora de reconocer las diferentes características y comportamientos que pueden llegar a desarrollar estos colectivos.

En efecto, la posibilidad de que los responsables de marketing puedan abordar a una subcultura de consumo como si fuera una comunidad de marca, puede resultar en nefastas consecuencias para cuestiones como la estabilidad y la lealtad de la propia marca. Del mismo modo, podría ocurrir que colectivos como la tribu consumidora, no fueran atendidos de la manera correcta resultando en una pérdida de oportunidades debido a una potencial miopía a la hora de reconocer el vínculo emocional que destaca y parece capital entre sus miembros. En definitiva, parece de inestimable importancia para la aplicación de estrategias de branding entre los profesionales, distinguir entre los diferentes tipos de agrupaciones y reconocer la coexistencia de las características de los sujetos que los integran.

Asimismo, se ha observado que la comunidad científica con respecto al estudio del comportamiento del consumidor colectivo y las marcas es un tema ampliamente abordado por las revistas norteamericanas y anglosajonas, algo que, si bien es frecuente en otras materias y para otras áreas, pone de manifiesto la necesidad de contar con más trabajos que aporten perspectivas alternativas. En esta línea y como posibles futuras líneas de investigación, por un lado, se plantea la posibilidad de realizar estudios empíricos que pongan en práctica la aplicación de estos conceptos en grupos de consumidores concretos.

Por otro lado y dado que esta investigación también advierte la importancia que el estudio de estos sujetos pudiera tener para otras disciplinas como la psicología. Partiendo de que la pertenencia a estas agrupaciones se observa determinante en el

Vivat Academia. Revista de Comunicación. 15 junio 2020 /15 septiembre 2020, nº 151, 69-83 
Sanz-Marcos, P. y Elías-Zambrano, R.

La experiencia comunitaria del consumo de marcas. De la subcultura de consumo a la tribu consumidora

comportamiento del consumidor, se pueden abrir futuras líneas de investigación que consigan vincular la conducta social de los individuos y sus posibles implicaciones para el marketing.

En definitiva, el poder de las comunidades en el mercado no puede ser subestimado debido a que los vínculos sociales entre los sujetos proporcionan valor y recursos importantes que permiten a los individuos construir su identidad, así como influir en las elecciones de consumo de los demás.

\section{REFERENCIAS}

Bazaki, E. \& Veloutsou, C. (2010). Brand communities, subcultures of consumption, neotribes: a melange of terminology, en G. Chrisodoulides, et al. (Eds.). Contemporary Issues in Brand Research (pp. 163-180). Atenas: Institute for Education and Research.

Biraghi, S; Gambetti, R. \& Pace, S. (2018). Between tribes and markets: The emergence of a liquid consumer-entrepreneuship. Journal of Business Research, (92), 392-402.

Canniford, R. (2011). A Typology of Consumption Communities. Research in Consumer Behavior, (13), 57-75. Recuperado de https://tinyurl.com/y7kzhpgcw

Cova, B. (1997). Community and consumption: Towards a definition of the «linking value» of product or services. European Journal of Marketing, 31(3), 297-316. doi: https://doi.org/10.1108/03090569710162380

Cova, B. (1999). Tribal marketing: A Latin deconstruction of a northern construction. First International Conference on Critical management Studies, UMIST.

Cova, B. \& Cova, V. (2002). Tribal marketing: The tribalisation of society and its impact on the conduct of marketing. European Journal of Marketing, 36(5/6), 595620. doi: https://doi.org/10.1108/03090560210423023

Cova, B. \& Dalli, D. (2008). From communal resistance to tribal value creation. 1st International Conference on Consumption and Consumer Resistance, Paris.

Dahl, S. (2014). Social Media Marketing-Theories and Applications. London: Sage.

De Burgh-Woodman, H. \& Brace-Govan, J. (2007). We do not live to buy: Why subcultures are different from brand communities and the meaning for marketing discourse. International Journal of sociology and social Policy, 27(5/6), 193-207. doi: https://doi.org/10.1108/01443330710757230

Drawbaugh, K. (2001). Las marcas a examen. El gran desafio de la identidad comercial. Madrid: Prentice Hall. 
Sanz-Marcos, P. y Elías-Zambrano, R.

La experiencia comunitaria del consumo de marcas. De la subcultura de consumo a la tribu consumidora

Fernández Gómez, J. D. (2013). Principios de estrategia publicitaria y gestión de marcas. Nuevas tendencias de brand management. Madrid: McGraw-Hill.

Firat, F. \& Venkatesh, A. (1993). Postmodernity: the age of marketing. International, en Journal of Research in Marketing, 10(3), 227-49.

Firat, F.; Dholakia, N. \& Venkatesh, A. (1995). Marketing in a postmodern world. European Journal of Marketing, 29(1), 40-56.

Goulding, C.; Shankar, A \& Canniford, R. (2013). Learning to be tribal: facilitating the formation of consumer tribes. European Journal of Marketing, 47(5/6), 813-832. doi: https://doi.org/10.1108/03090561311306886

Kett, A. (1999). Subcultures or Neotribes: Rethinking the Relationship Between Youth, Style and Musical Tastes. Sociology, (33), 599-614.

Kozinets, R. (1999). E-Tribalized Marketing?: The Strategic Implications of Virtual Communities of Consumption. European Management Journal, 17(3), 252-264.

Mamali, E; Nuttall, P. \& Shankar, A. (2018). Formalizing consumer tribes: Towards a theorization of consumer-constructed organizations. Marketing Theory, 18(8), 521542.

McAlexander, J.; Schouten, J. \& Koenig, H. (2002). Building Brand Community. Journal of Marketing, (66), 38-54.

Muñiz, A. \& O'Guinn, T. (2001). Brand Community. Journal of Consumer Research, 27(4), 412-432. doi: https:// doi.org/10.1086/319618

Närvänen, E.; Kartastenpää, E., \& Kuusela, H.(2013). Online lifestyle consumption community dynamics: A practice-based analysis. Journal of Consumer Behaviour, 12(5), 358 369. doi: https:// doi.org/10.1002/cb.1433

Richardson, B. (2013). Tribal Marketing, Tribal Branding. An expert guide to the brand co creation process. UK: Palgrave Macmillan.

Saat, R.; Maisurah, K. \& Hanim, R. (2015). Developing Tribe Marketing and Information Technology Competencies Skills (Tribe-IT Skills) for Virtual Entrepreneurs. Proceedings of the International Conference on E-Commerce.

Schouten, J. \& Mcalexander, J. (1995). Subcultures of Consumption: An Ethnography of the New Bikers. Journal of Consumer Research, 22(1), 43-61. Recuperado de https://www.jstor.org/stable/2489699

Silva, S. \& Santos, M. (2012). How to capitalise on a tribe. The Marketing Review, 12(4), 417-434. https:// doi.org/10.1362/146934712X13469451716718

Vivat Academia. Revista de Comunicación. 15 junio 2020 /15 septiembre 2020, nº 151, 69-83 
Sanz-Marcos, P. y Elías-Zambrano, R.

La experiencia comunitaria del consumo de marcas. De la subcultura de consumo a la tribu consumidora

Taute, H. \& Sierra, J. (2014). Brand tribalism: an anthropological perspective, Journal of Product \& Brand Management, 23(1), 2-15.

Thomas, T.; Price, L. \& Schau, H. (2013). When Differences Unite: Resource Dependence in Heterogeneous Consumption Communities. Journal of Consumer Research, 39(5), 1010-1033. doi: https://doi.org/10.1086/666616

Thompson, C. \& Troester, M. (2002). Consumer Value Systems in the Postmodern Fragmentation: The Case of the Natural Health Microculture. Journal of Consumer Research, (28), 550-571.

\section{AUTORES:}

\section{Paloma Sanz-Marcos}

Es profesora del departamento de Comunicación audiovisual y Publicidad de la Universidad de Sevilla (acreditada a contratado doctor por la ANECA). Doctora en Comunicación (mención internacional por la UC Berkeley, California) y licenciada en Publicidad y Relaciones Públicas, compagina sus labores docentes con la investigación en el área de la comunicación. Miembro del grupo de investigación IDECO, ha presentado comunicaciones en diversos encuentros académicos y ha publicado artículos y capítulos de libros sobre publicidad y brand management. Ha sido profesora visitante en universidades extranjeras como UC Berkeley, Universidade do Algarve (Portugal) o la Pontificia Universidad Católica de Chile. palomasanz@us.es

Orcid ID: http://orcid.org/0000-0002-6103-6993

\section{Rodrigo Elías-Zambrano}

Licenciado en Comunicación AV, Máster en Gestión de Empresas AV y Experto en ELearning por US, y Máster Oficial en Comunicación y Educación AV por la Universidad de Huelva, es Doctor por Universidad de Sevilla. Ha publicado diversos artículos relacionados con las estrategias de comunicación, la publicidad comercial y la comunicación audiovisual y su relación con la educación. Es miembro del Grupo de investigación SEJ420.

rodrigoelias@us.es

Orcid ID: $\underline{\text { http:/ / orcid.org/0000-0001-8256-582X }}$ 